\title{
Microhabitat de Habia rubica (Vieillot) e Trichothraupis melanops (Vieillot) (Aves, Emberizidae, Thraupinae), em uma floresta atlântica do sul do Brasil
}

\author{
Fernando de Lima Fávaro ${ }^{1} \&$ Luiz dos Anjos ${ }^{1,2}$ \\ 1 Programa de Pós-Graduação em Ciências Biológicas, Universidade Estadual de Londrina. Caixa Postal 6001, 86051-990 \\ Londrina, Paraná, Brasil. E-mail: flfavaro@uel.br \\ 2 Departamento de Biologia Animal e Vegetal, Universidade Estadual de Londrina. Caixa Postal 6001, 86051-990 Londrina, \\ Paraná, Brasil.
}

\begin{abstract}
Microhabitat of Habia rubica (Vieillot) and Trichothraupis melanops (Vieillot) (Aves, Emberizidae, Thraupinae) in an Atlantic Forest, Southern Brazil. This study identifies which variables characterize the microhabitats of Habia rubica (Vieillot, 1817) and Trichothraupis melanops (Vieillot, 1818) and verifies the pattern of abundance of these species in relation to the parameters of microhabitat. Principal components analysis showed the variables of microhabitat more important for each species. The microhabitat of $H$. rubica was characterized for the middle stratum open and understory unclear, rich in ferns, young trees, herbs and lianes; this species presented higher abundance in places rich in such variables. For $T$. melanops, the variables more important were understory unclear with ferns, shrubs, young trees, lianes and herbs, large trees and number of trees with bryophytes; the abundance of this species was unrelated with the density of these variables perhaps due to their generalist habits.
\end{abstract}

KEY WORDS. Forest fragmentation, microhabitat, Paraná.

RESUMO. Este estudo identifica quais variáveis caracterizam os microhabitats de Habia rubica (Vieillot, 1817) e Trichothraups melanops (Vieillot, 1818) e verifica o padrão de abundância dessas espécies em relação aos parâmetros de microhabitat. Análise de componentes principais mostrou as variáveis de microhabitat mais importantes para cada espécie. $\mathrm{O}$ microhabitat de $H$. rubica foi caracterizado pelo estrato médio aberto e sub-bosque denso e rico em samambaias, arvoretas, herbáceas e cipós; esta espécie apresentou maior abundância nos locais ricos em tais variáveis. Para $T$. melanops, as variáveis mais importantes foram samambaias, arbustos, arvoretas, herbáceas, cipós, árvores de grande porte e quantidade de árvores com briófitas; a abundância desta espécie não foi relacionada com a densidade dessas variáveis talvez devido aos seus hábitos generalistas.

PALAVRAS CHAVES. Fragmentação florestal, microhabitat, Paraná.

O processo de ocupação do Brasil levou o bioma Floresta Atlântica a uma drástica redução de sua cobertura vegetal original, hoje disposta esparsamente ao longo da costa brasileira e no interior das regiões Sul e Sudeste, além de importantes fragmentos no sul dos estados de Goiás e Mato Grosso do Sul e no interior dos estados do Nordeste (Fundação S.O.S. Mata AtlânTICA 1998). Atualmente no Brasil, áreas de floresta contínua estão concentradas na região amazônica, embora estas também estejam sofrendo intensa devastação, em função de atividades econômicas e de desenvolvimento sócio-regional.

No norte do Estado do Paraná, a atividade agrícola iniciada a partir de 1920 reduziu a floresta contínua a fragmentos florestais pequenos e esparsos (ANjos 1998). Dentre os poucos remanescentes florestais, merece destaque o Parque Estadual Mata dos Godoy (656 ha), considerado o principal remanes- cente de floresta estacional semi-decidual do norte do Paraná (F́́varo \& STipp 2003).

A ausência de determinadas espécies de aves em fragmentos florestais é devida à perda ou alteração da heterogeneidade vegetal, (BierregaArd 1990, Anjos \& Boçon 1999, Gimenes \& AnJos 2000), a qual define a ocorrência de microhabitats, isto é, habitats que apresentam aspectos distintos dos demais e que, por isso, são críticos para certas espécies de aves (Sтотz et al. 1996). A presença e a distribuição de uma determinada espécie em um local estariam relacionadas à ocorrência de microhabitats (Guido \& GianelLe 2001).

Thraupinae (Emberizidae) é constituída por aves neotropicais que são encontradas em diversos ambientes, desde planícies tropicais até regiões temperadas nos Andes, onde atinge maior diversidade (Meyer de Schauensee 1970, Ridgely \& Tudor 
1989). As espécies chamam a atenção pelo exuberante colorido de suas plumagens, o que as colocam entre as mais bonitas aves neotropicais. A alimentação é constituída de pequenos artrópodes e frutos (Sick 1997). Assim como outras aves, os traupíneos freqüentemente juntam-se em bandos mistos para procurar alimento, nas copas das árvores ou no interior das florestas (Meyer de SCHAuensee 1970).

Habia rubica (Vieillot, 1817) apresenta plumagem vermelho-apagada, com exceção do píleo que é escarlate reluzente, enquanto a fêmea é parda (Ridgely \& Tudor 1989, SiCK 1997). Habita o interior de florestas, normalmente em pares ou em bandos mistos (Sick 1997). Alimenta-se de pequenos frutos e, preferencialmente, de insetos. Emite gritos para advertir os inimigos sendo, por isso, considerada espécie nuclear, atuando como sentinela e, dessa forma, contribuindo na formação e na manutenção da coesão de bandos mistos (Develey 2001).

Trichothraupis melanops (Vieillot, 1818) caracteriza-se por apresentar o píleo amarelado contrastando com a face negra. $\mathrm{O}$ dorso é cinzento, com asas e cauda negras. A fêmea apresenta-se sem o negro na face e com pouco amarelo no píleo (SICK 1997, Ridgely \& Tudor 1989). Habita o interior das florestas densas e secundárias, a procura de insetos. Geralmente em pares ou pequenos grupos mistos, também atuando como espécie nuclear (Develey 2001). Rodrigues et al. (1994) descreveram que esta espécie, além apresentar associação com bandos mistos de outras aves e com formigas de correição, também apresentou relação com duas espécies de macacos para a obtenção de alimento: Cebus apella (Linnaeus, 1758) e Alouatta fusca (É. Geoffroy, 1812).

O objetivo foi analisar quais variáveis ambientais caracterizam os microhabitats de H. rubica e T. melanops e, também, a relação entre a abundância dessas espécies e a presença das variáveis ambientais que caracterizam seus microhabitats.

\section{MATERIAL E MÉTODOS}

\section{Área de estudo}

O Parque Estadual Mata dos Godoy $\left(23^{\circ} 27^{\prime} \mathrm{S}, 51^{\circ} 15^{\prime} \mathrm{W}\right)$ está localizado a cerca de 15 quilômetros ao sul da cidade de Londrina, Estado do Paraná (Fig. 1). É o maior e mais bem preservado remanescente de floresta estacional semi-decidual do estado, com 656 ha (FÁvARo \& STIPP 2003). Esse parque encontra-se circundado por áreas cultivadas e é delimitado, ao sul, pelo Ribeirão dos Apertados, afluente do Rio Tibagi.

Na porção sul, a altitude é de $500 \mathrm{~m}$ e dentre as espécies arbóreas, as mais abundantes são Nectandra megapotamica Mez (Lauraceae), Campomanesia xanthocarpa O. Berg (Myrtaceae), Actinostemon concolor Müll. Arg. (Euphorbiaceae) e Parapiptadenia rigida Brenan (Mimosoidae, Soares-silva et al. 1998). Na borda do Ribeirão dos Apertados há uma faixa de terra não inclinada, de 10 a 30 m de extensão, que é periodicamente alagada (BIANCHINI et al. 2001, 2003).

A porção norte do parque possui uma altitude de $650 \mathrm{~m}$ (Silva \& Soares-Silva 2000), onde a vegetação arbórea do subbosque, na maioria, não ultrapassa $8 \mathrm{~m}$ (SOAREs-Silva \& BARRoso
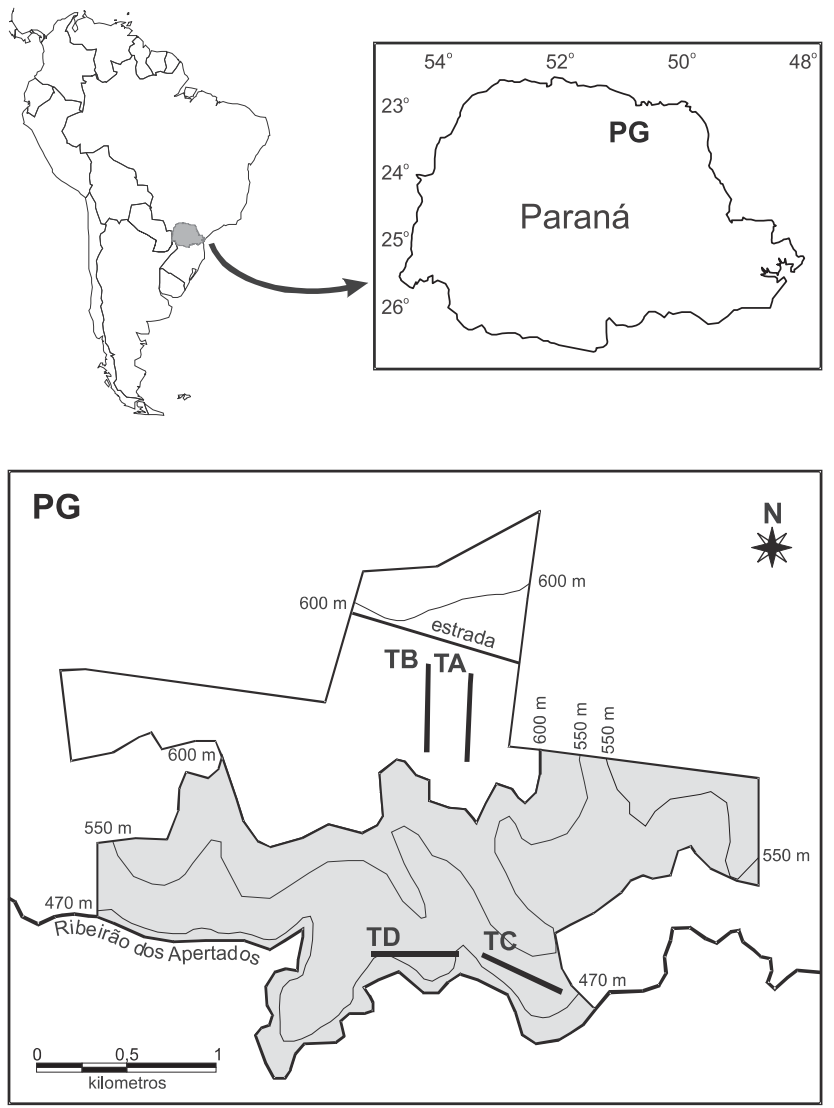

Figura 1. Parque Estadual Mata dos Godoy, mostrando as transecções da porção sul (C e D) e da porção norte (A e B).

1992), como é o caso de Trichilia elegans A. Juss. (Meliaceae) e A. concolor. Dentre as es espécies emergentes, com mais de 40 m, estão Aspidosperma polyneuron Müll. Arg. (Apocynaceae) e Ficus insipida Willd. (Moraceae, SoAres-Silva \& Barroso 1992, Silva \& SoARes-Silva 2000). Ocupando uma posição intermediária entre o sub-bosque e o dossel estão Cabralea canjerana Mart. (Meliaceae) e N. megapotamica, entre outras.

Quatro transecções foram abertas, sendo duas em cada porção (Fig. 1). Essas transecções tinham comprimento de 500 metros e em cada uma delas foram estabelecidos cinco pontos, distantes $100 \mathrm{~m}$ um do outro. O primeiro ponto encontrava-se a 100 m de distância da entrada da transecção.

\section{Procedimentos de campo}

A coleta de dados foi realizada mensalmente entre setembro e dezembro de 2002. O número de contatos foi estimado pelo método de amostragem por pontos de escuta (BLONDEL et al. 1970, Vielliard \& Silva 1990). O tempo de amostragem em cada ponto foi de 20 minutos. As amostragens começaram ao amanhecer no primeiro ponto e encerraram após cerca de três horas no último ponto. O uso de uma ficha de campo padronizada, dividida em quadrantes, evitou que um mesmo indiví- 
duo ou grupo fosse contado mais de uma vez. Ao final dos quatro meses foram realizadas 16 amostragens, totalizando 48 horas esforço de amostragem.

A caracterização de microhabitats foi realizada de duas maneiras: após o encerramento de cada amostragem quantitativa e em dias destinados apenas para a caracterização. As transecções foram percorridas lentamente e em cada ponto foi feita uma parada de cinco minutos para que as espécies, caso estivessem vocalizando, fossem localizadas. Espécies localizadas durante o deslocamento entre os pontos também foram consideradas. A partir do ponto onde o animal se encontrava foi estabelecido um raio de $10 \mathrm{~m}$, dentro do qual a coleta de informações sobre as variáveis ambientais foi realizada.

A definição das variáveis ambientais foi feita a partir de visitas prévias ao local de estudo, onde foram realizadas as primeiras observações das espécies e de seus habitats. Oito variáveis ambientais foram definidas:samambaias, arbustos, arvoretas, herbáceas, emaranhados de cipós e galhos, árvores de grande porte, quantidade de árvores com briófitas e estrato médio.

Tais variáveis foram analisadas, dentro de uma escala $(0$, 1 ou 2). Para as variáveis samambaias, arbustos, arvoretas, herbáceas e emaranhados, a escala 0 indica ausência, 1 indica presença em até $10 \%$ da área em torno do local onde o animal estava e 2 indica presença em mais de $10 \%$ da área. Quanto às árvores de grande porte, 0 indica ausência, 1 indica presença de até três árvores e 2 indica presença de mais de três árvores. Para quantidade de árvores com briófitas 0 indica ausência de briófitas, 1 presença em até 50\% das árvores e 2 presença em mais de $50 \%$. Para estrato médio 0 indica que o dossel é visto entre as copas das árvores, 1 indica que o dossel é visto entre a folhagem de cada árvore e 2 o dossel não é visto, devido à densidade da folhagem.

Como o parque apresenta diferenças na estrutura vegetacional, optou-se por analisar separadamente as porções sul e norte, de modo que uma porção servisse de parâmetro de com- paração com a outra.

\section{Análise dos dados}

Através do método de amostragem por pontos de escuta foi obtido um valor de abundância de cada espécie a partir do número de contatos nos pontos de amostragem. A abundância é expressa na forma de um índice, denominado Índice Pontual de Abundância (IPA), que é calculado dividindo-se o número total de contatos da espécie pelo número de pontos amostrados.

Para saber quais variáveis ambientais foram as mais importantes, isto é, as que melhor identificavam os habitats ocupados pelas espécies, os dados obtidos foram correlacionados através da Análise de Componentes Principais (ACP), utilizando o software Statistica Version 5. Diferenças no número de contatos foram testadas através de qui-quadrado.

\section{RESULTADOS}

Foram obtidos 31 registros para H. rubica (18 na porção sul e 13 na porção norte). Na ACP da porção sul, os fatores 1 e 2 explicaram 55,33\% da variância dos dados obtidos e as variáveis mais representativas foram quantidade de árvores com briófitas, arvoretas, estrato médio, samambaias, herbáceas e emaranhados. Para a porção norte, os fatores 1 e 2 explicaram $49,52 \%$ da variância dos dados obtidos e as variáveis mais representativas foram herbáceas, arvoretas, quantidade de árvores com briófitas e emaranhados (Tab. I).

Para T. melanops foram obtidos 34 registros (11 na porção sul e 23 na porção norte). Na ACP da porção sul, os fatores 1 e 2 explicaram $64,37 \%$ da variância dos dados obtidos e as variáveis mais representativas foram arbustos, samambaias, herbáceas, arvoretas, emaranhados e árvores de grande porte. Para a porção norte, os fatores 1 e 2 explicaram 53,79\% da variância dos dados obtidos e as variáveis mais representativas foram árvores de grande porte, herbáceas, samambaias, arvoretas e quantidade de árvores com briófitas (Tab. I).

Tabela I. Análise de Componentes Principais para Habia rubica nas e Trichothraupis melanops porções sul e norte do Parque Estadual Mata dos Godoy.

\begin{tabular}{|c|c|c|c|c|c|c|c|c|}
\hline \multirow{3}{*}{ Variáveis } & \multicolumn{4}{|c|}{ Habia rubica } & \multicolumn{4}{|c|}{ Trichothraupis melanops } \\
\hline & \multicolumn{2}{|c|}{ Porção Sul } & \multicolumn{2}{|c|}{ Porção Norte } & \multicolumn{2}{|c|}{ Porção Sul } & \multicolumn{2}{|c|}{ Porção Norte } \\
\hline & Fator 1 & Fator 2 & Fator 1 & Fator 2 & Fator 1 & Fator 2 & Fator 1 & Fator 2 \\
\hline Samambaias & $0,66^{*}$ & $-0,06$ & 0,47 & $-0,12$ & $0,81^{*}$ & 0,21 & $-0,73^{*}$ & 0,29 \\
\hline Arbustos & ** & ** & $-0,22$ & $-0,53$ & $0,90^{*}$ & $-0,20$ & $-0,57$ & $-0,24$ \\
\hline Arvoretas & 0,15 & $0,76^{*}$ & $0,76^{*}$ & $-0,11$ & $0,75^{*}$ & $-0,15$ & $-0,69^{*}$ & $-0,25$ \\
\hline Herbáceas & $0,65^{*}$ & $-0,51$ & $0,79 *$ & $-0,11$ & $0,76^{*}$ & 0,03 & $-0,79^{*}$ & 0,13 \\
\hline Emaranhados & $0,64^{*}$ & $-0,37$ & $-0,62^{*}$ & 0,07 & $-0,03$ & $0,72^{*}$ & 0,60 & $-0,17$ \\
\hline Árvores de grande porte & 0,55 & 0,35 & 0,51 & 0,43 & 0,28 & $0,72^{*}$ & $-0,17$ & $0,84^{*}$ \\
\hline Quantidade de árvores com briófitas & 0,33 & $0,77^{*}$ & 0,40 & $0,70^{*}$ & $0,69^{*}$ & $-0,53$ & $-0,01$ & $0,68^{*}$ \\
\hline Estrato médio & $-0,70^{*}$ & $-0,06$ & $-0,58$ & 0,59 & $-0,59$ & $-0,46$ & 0,59 & 0,43 \\
\hline Variância acumulada (\%) & 31,35 & 55,33 & 32,61 & 49,52 & 43,81 & 64,37 & 33,76 & 53,79 \\
\hline
\end{tabular}

${ }^{*}$ ) Variáveis que melhor identificam o habitat; $\left.{ }^{* *}\right)$ não apresentou variância. 
Quanto à abundância, $H$. rubica apresentou um IPA maior nas transecções do sul $(\mathrm{C}+\mathrm{D}=1,15)$ em relação ao norte (A $+B=0,20)$. A diferença entre o número de contatos da porção sul $(\mathrm{C}+\mathrm{D}=23)$ e o da porção norte $(\mathrm{A}+\mathrm{B}=4)$ mostrou-se significativa $\left(\mathrm{c}^{2}=13,38 ; \mathrm{gl}=1 ; \mathrm{p}<0,001\right)$.

Trichothraupis melanops apresentou IPAs semelhantes nestas transecções $(C+D=0,70 ; A+B=0,95)$. Não houve diferença significativa $\left(\chi^{2}=0,758 ; \mathrm{gl}=1 ; \mathrm{p}>0,05\right)$ entre o número de contatos da porção sul $(C+D=14)$ e da porção norte $(A+B=19)$.

\section{DISCUSSÃO}

Microhabitats são importantes por definirem os padrões de distribuição e de abundância dos organismos (Sтотz et al. 1996, Guido \& Gianelle 2001). Na porção sul, as variáveis quantidade de árvores com briófitas e estrato médio são importantes para $H$. rubica, sugerindo a ocorrência de locais sombrios nesta porção do parque. As variáveis samambaias, herbáceas e arvoretas, da mesma forma, sugerem um ambiente pouco iluminado. Essa característica coincide com a descrita por SicK (1997) e Narosky \& IzURIETA (2003), na qual H. rubica habita ambientes sombrios no interior de florestas. A presença de emaranhados de cipós e galhos também sugere que $H$. rubica tenha preferência por ambientes mais densos, talvez para se proteger de possíveis predadores. Na porção norte do parque, o menor número de variáveis representativas pode ser devido à própria configuração vegetacional desta porção que, naturalmente, impossibilita a ocorrência de habitats mais amplos.

Quanto a T. melanops, todas as variáveis ambientais mostraram-se importantes para a espécie na porção sul, exceto estrato médio, enquanto que na porção norte, cinco variáveis se destacaram. Em ambas as porções do parque, T. melanops teve relação com mais variáveis do que $H$. rubica, o que sugere que T. melanops apresenta uma potencialidade para explorar ambientes mais diversificados. Rodrigues (1995), analisando a sobreposição espacial e de nicho alimentar entre diversas espécies de traupíneos, destaca flexibilidade de T. melanops em explorar diferentes tipos de substratos, principalmente no subbosque.

Ambas as espécies utilizaram uma quantidade maior de variáveis na porção sul do parque em relação à porção norte. É possível que isto esteja relacionado à forte declividade presente na porção sul a qual estaria proporcionando um número maior de habitats para essas espécies. O número de contatos semelhante de T. melanops nas duas porções do parque coincide com o fato de essa espécie ter tido relação com uma maior quantidade de variáveis em ambas as porções. Desse modo, é provável que T. melanops seja uma espécie mais generalista que H. rubica, em relação aos ambientes explorados. A significativa presença de $H$. rubica na porção sul pode ser devido ao fato de seu ambiente preferencial (uma vegetação densa e com pouca incidência de luz) estar fortemente representado nesta porção do parque.

\section{AGRADECIMENTOS}

Patrícia P. Serafini, Fabíola Poletto, Edson V. Lopes e Graziele H. Volpato auxiliaram nas atividades de campo. Os professores José A. Pimenta e José H.F. Mariño fizeram correções e sugestões ao trabalho de conclusão do curso de graduação em Ciências Biológicas da Universidade Estadual de Londrina, do qual originou este estudo. Lorenzo Zanetti auxiliou na análise estatística dos dados. O Instituto Ambiental do Paraná (IAP) concedeu autorização para os trabalhos de campo no Parque Estadual Mata dos Godoy. CNPq forneceu apoio financeiro, dentro do Programa Mata Atlântica (Assessoria de Cooperação Internacional, processo 690146/01-9). O segundo autor recebe Bolsa Produtividade Científica do CNPq (350054/95-9).

\section{REFERÊNCIAS BIOBLIOGRÁFICAS}

ANJos, L. Dos. 1998. Conseqüências biológicas da fragmentação no norte do Paraná. IPEF, Curitiba, 12 (32): 87-94.

Anjos, L. Dos \& R. Boçon. 1999. Bird communities in natural forest patches in southern Brazil. The Wilson Bulletin, Lawrence, 111 (3): 397-414.

Bianchini, E.; J.A. Pimenta \& F.A.M. dos Santos. 2001. Spatial and temporal variation in the canopy cover in a tropical semideciduous forest. Brazilian Archives of Biology and Technology, Curitiba, 44 (3): 269-276.

Bianchini, E.; R.S. Popolo; M.C. Dias \& J.A. Pimenta. 2003. Diversidade e estrutura de espécies arbóreas em área alagável do município de Londrina, sul do Brasil. Acta Botanica brasilica, São Paulo, 17 (3): 405-419.

BierRegaARd JR., R.O. 1990. Avian communities in the understory of Amazonian forest fragments, p. 333-343. In: A. KEAST. (Ed.). Biogeography and ecology of forest bird communities. The Hague, SPB Academic Publishing, 410p.

Blondel, J.; C. Ferry \& B. Frochot. 1970. La méthode des indices ponctuels d'abondance (I.P.A.) ou des relevés d'avifaune par "stations d'écoute". Alauda, Paris, 38: 55-71.

Develey, P.F. 2001. Os bandos mistos de aves na florestas neotropicais, p. 39-47. In: J.L. B. AlbuQUERQUE; J.F. CÂNDIDOJúnior; F.C. Straube \& A.L. Roos (Eds). Ornitologia e conservação: da ciência às estratégias. Tubarão, Editora da Universidade do Sul de Santa Catarina, 344p.

FÁvARo, F.L. \& N.A.F. STIPP. 2003. Análise dos remanescentes florestais da bacia do ribeirão dos Apertados, a partir de imagens LandSat. Geografia, Londrina, 12 (2): 91-105.

Fundação S.O.S. Mata Atlântica.1998. Atlas da evolução dos remanescentes florestais e ecossistemas associados no domínio da Mata Atlântica no período 1990-1995. São Paulo, Fundação S.O.S. Mata Atlântica, Instituto Nacional de Pesquisas Espaciais, Instituto Sócio-ambiental, 55p.

Gimenes, M.R. \& L. Dos Anjos. 2000. Distribuição espacial de aves em um fragmento florestal do Campus da Universidade Estadual de Londrina, Norte do Paraná, Brasil. Revista Brasileira de Zoologia, Curitiba, 17 (1): 263-271.

Revista Brasileira de Zoologia 22 (1): 213-217, março 2005 
Guido, M. \& D. Gianelle. 2001. Distribution patterns of four Orthoptera species in relation to microhabitat heterogeneity in an ecotonal area. Acta Oecologica, Paris, 22: 175-185.

Meyer de Schauensee, R. 1970. A guide to the birds of South America. Philadelphia, The Academy of Natural Sciences, $500 \mathrm{p}$.

NARosky, T. \& D. YzURIETA. 2003. Guía para la identificación de las aves de Argentina y Uruguay. Buenos Aires, Vazquez Mazzini, $15^{\mathrm{a}}$ ed., 348p.

Ridgely, R.S. \& G. Tudor. 1989. The birds of South America. The Oscine Passerines. Austin, University of Texas Press, vol. 1, 518p.

Rodrigues, M. 1995. Spatial distribution and food utilization among tanagers in southeastern Brazil (Passeriformes: Emberizidae). Ararajuba, Rio de Janeiro, 3: 27-32.

Rodrigues, M.; C.G. Machado; S.M.R. Alvares \& M. Galetti. 1994. Association of the Black-Googgled Tanager (Trichothraupis melanops) with Flushers. Biotropica, Washington, 26 (4): 472-475.

SICK, H. 1997. Ornitologia brasileira. Rio de Janeiro, Nova Fron- teira, 912p.

SiLVA, F.C. \& L.H. SoARES-SiLVA. 2000. Arboreal flora of the Godoy Forest State Park, Londrina, PR., Brazil. Edinburgh Journal of Botany, Edinburgh, 57 (1): 107-120.

Soares-Silva, L.H. \& G.M. Barroso. 1992. Fitossociologia do estrato arbóreo da floresta na porção norte do Parque Estadual Mata dos Godoy, Londrina-PR, Brasil. In: Anais do $\mathbf{8}^{\circ}$ Congresso SBPC, Campinas, p. 101-112.

Soares-Silva, L.H.; K.K. Kita \& F.C. Silva. 1998. Fitossociologia de um trecho de floresta de galeria no Parque Estadual Mata dos Godoy, Londrina, PR, Brasil. Boletim do Herbário Ezechias Paulo Heringer, Brasília, 3: 46-62.

Stotz, D. F.; J.W. Fitzpatrick; T.A. Parker III \& D.K. Moskovits.1996. Neotropical birds: ecology and conservation. Chicago, The University of Chicago Press, 481p.

Vielliard, J.E.M. \& W.R. Silva. 1990. Nova metodologia de levantamento quantitativo da avifauna e primeiros resultados no interior do Estado de São Paulo, Brasil. In: Anais do IV Encontro Nacional dos Anilhadores de Aves, Recife, p. 117-151.

Recebido em 27.IV.2004; aceito em 18.II.2005. 